PROCEEDINGS OF THE

AMERICAN MATHEMATICAL SOCIETY

Volume 129, Number 2, Pages 581-584

S 0002-9939(00)05599-4

Article electronically published on August 28, 2000

\title{
A MODEL FORM FOR EXACT $b$-METRICS
}

\author{
M. S. JOSHI
}

(Communicated by Józef Dodziuk)

\begin{abstract}
Any manifold with boundary can be equipped with a $b$-metric which takes the form $\frac{d x^{2}}{x^{2}}+h(x, y, d x, d y)$ with respect to some product decomposition near the boundary, and $h$ positive definite on restriction to the tangent space of the boundary. Here we show the existence of a product decomposition such that $h$ is independent of $d x$ modulo terms vanishing to infinite order at the boundary. The uniqueness of this decomposition is also examined.
\end{abstract}

\section{INTRODUCTION}

Scattering theories have recently been developed for compact manifolds with various sorts of complete metrics. In particular there has been interest in manifolds with asymptotically hyperbolic ends or zero-metrics, asymptotically Euclidean ends or scattering metrics, and asymptotically cylindrical ends or $b$-metrics. For a discussion of these cases, we refer the reader to Melrose's overview, [4]. In this note, we show that techniques developed to prove the existence of model forms for zero and scattering metrics, [2], 3], can also be applied to $b$-metrics. As well as simplifying the analysis, these model forms are crucial if one wishes to prove inverse results. For a discussion of scattering on manifolds with $b$-metrics we refer the reader to [1] and for a general discussion of analysis on manifolds with $b$-metrics to 5 .

Let $(X, \partial X)$ be a compact manifold with boundary. An exact $b$-metric is a smooth metric in the interior of $X$ which for some boundary defining function $x$ takes the form

$$
g=\frac{d x^{2}}{x^{2}}+g^{\prime}
$$

where $g^{\prime}$ is a smooth 2-cotensor which is smooth up to the boundary and its restriction, $h$, to the boundary is positive definite on the tangent space of the boundary.

If we take a product decomposition of some open neighbourhood, $U$, of the boundary,

then we can write

$$
p \mapsto(x, y) \in[0, \epsilon) \times \partial X,
$$

$$
g=\frac{d x^{2}}{x^{2}}+g^{\prime}(x, y, d x, d y) .
$$

An interesting question is whether there exists a product decomposition such that $g^{\prime}$ is independent of $d x$. From the results of [2], [3, one would expect this to be the

Received by the editors April 15, 1999.

2000 Mathematics Subject Classification. Primary 58J50.

Key words and phrases. Scattering theory, model, $b$-metric.

(C) 2000 M. S. Joshi 
case. Here we show the existence of such a product decomposition modulo an error vanishing to infinite order at the boundary.

We also show that the boundary defining function is determined up to a scalar constant, modulo terms vanishing to infinite order at the boundary, by the metric and the smooth structure.

It is likely that there exists a product decomposition which removes the order infinity error but it is not clear how to proceed and we leave this to other authors.

\section{Construction of the model}

In this section, we prove our model theorem and then discuss its rigidity. We prove the model theorem by constructing a sequence of diffeomorphisms which approximate to succeeding levels of precision and then apply the Borel Lemma to obtain our final diffeomorphism.

Theorem 2.1. Let $(X, \partial X)$ be a compact manifold with boundary. Let $g$ be an exact $b$-metric on $X$. Then there exists a diffeomorphism,

$$
\psi:[0, \epsilon) \times \partial X \rightarrow U
$$

with $U$ a neighbourhood of the boundary such that

$$
\psi^{*} g=\frac{d x^{2}}{x^{2}}+h(x, y, d y)+\mathcal{O}\left(x^{\infty}\right) .
$$

Proof. Picking an arbitrary product decomposition (which exists by taking geodesic normal coordinates with respect to a metric which is smooth up to the boundary) and using our definition of a $b$-metric we have the existence of a diffeomorphism $\phi$ such that

$$
\phi^{*} g=\frac{d x^{2}}{x^{2}}+\gamma(x, y, d y)+\alpha(x, y) d x^{2}+\beta(x, y, d y) d x,
$$

with $\alpha, \beta, \gamma$ smooth up to $x=0$ and $\gamma(0, y, d y)$ positive definite.

Our objective is to eliminate $\alpha$ and $\beta$. We do this by computing a Taylor series term by term. We work purely in local coordinates on the boundary-we shall see that all choices are forced and so natural and there is therefore no problem with patching these local germs together.

Consider putting $x=X, y_{j}=Y_{j}+b_{j}(Y) X$. We then have

$$
\begin{aligned}
d x & =d X, \\
d y_{j} & =d Y_{j}+b_{j}(Y) d X+X \frac{\partial b_{j}}{\partial Y} d Y .
\end{aligned}
$$

If $\gamma(0, y, d y)=\sum_{i j} \gamma_{i j}(y) d y_{i} d y_{j}$, then the metric becomes

$$
\begin{aligned}
& \frac{d X^{2}}{X^{2}}+\sum_{i j} \gamma_{i j}(Y) d Y_{i} d Y_{j}+2 \sum_{i j} \gamma_{i j}(Y) b_{j} d Y_{i} d X+\sum_{i j} \gamma_{i j}(Y) b_{i} b_{j} d X^{2} \\
&+\tilde{\alpha}(X, Y) d X^{2}+\beta(X, Y, d Y) d X+\mathcal{O}(X) .
\end{aligned}
$$

As $\gamma_{i j}(y)$ is positive definite, there is a unique choice of $b_{j}$ such that

$$
\sum_{i j} \gamma_{i j}(Y) b_{j} d Y_{i} d X+\beta(0, Y, d Y) d X
$$

is equal to zero. We take this choice. 
We now have the metric in the form (2.1) with the added condition that $\beta(0, y, d y)$ $=0$. Now put $x=X+a(y) X^{3}, y=Y$. Then we have

$$
d x=d X+a(y) 3 X^{2} d X+\frac{\partial a}{\partial y} X^{3} d Y
$$

and the metric becomes

$$
\frac{d X^{2}}{X^{2}}+\gamma(X, Y, d Y)+4 a(y) d X^{2}+\tilde{\alpha}(X, Y) d X^{2}+\beta(X, Y, d Y) d X+\mathcal{O}(X) .
$$

So if we put $a=-\frac{1}{4} \tilde{\alpha}(0, y)$, we achieve the form (2.1) with both $\alpha$ and $\beta$ vanishing at $x=0$.

We have shown the existence of a diffemorphism $\psi_{0}$ of the form $\psi_{0}(X, Y)=$ $\left(X+a(Y) X^{3}, Y+b(Y) X\right)$ such that

$$
\psi_{0}^{*} \phi^{*} g=\frac{d X^{2}}{X^{2}}+h(X, Y, d Y)+X \alpha_{1}(X) d X^{2}+X \beta_{1}(X, Y, d Y) d X
$$

Now suppose we have constructed a sequence of diffeomorphisms, $\psi_{r}$, for $r<k$, such that

$$
\psi_{r}(X, Y)=\left(X+a_{r}(Y) X^{r+3}, Y+b_{r}(Y) X^{r+1}\right),
$$

and such that $\phi_{r}$, the composition of all the first $r$ maps with $\phi$, has the property

$$
\phi_{r}^{*} g=\frac{d x^{2}}{x^{2}}+\gamma_{r}(x, y, d y)+x^{r+1} a_{r}(x, y) d x^{2}+x^{r+1} b_{r}(x, y, d y) d x
$$

We now show how to construct $\phi_{k}$. As above, first we choose the next $Y$ term and then the next $X$ term.

So let $y=Y+X^{k+1} b_{k}, x=X$. The metric, $\phi_{k-1}^{*} g$, then becomes

$$
\begin{aligned}
& \frac{d X^{2}}{X^{2}}+\gamma_{k-1}(X, Y, d Y)+X^{k} \alpha_{k-1}(X, Y) d X^{2} \\
& \quad+X^{k} \beta_{k-1}(X, Y, d Y) d X+2(k+1) \sum_{i j} \gamma_{i j}(Y) b_{i, k} d Y_{j} d X+\mathcal{O}\left(X^{k+1}\right),
\end{aligned}
$$

where $b_{k}=\left(b_{1, k}, \ldots, b_{n-1, k}\right)$. As before there is then a unique choice of $b_{k}$ cancelling with $\beta_{k-1}$. We now have the metric in the form

$$
\frac{d x^{2}}{x^{2}}+\gamma_{k-1}(x, y, d y)+x^{k} \alpha_{k-1}(x, y) d x^{2}+\mathcal{O}\left(x^{k+1}\right) .
$$

Putting $x=X+X^{k+3} a_{k}, y=Y$, the metric becomes

$\frac{d X^{2}}{X^{2}}+\gamma_{k-1}(X, Y, d Y)+X^{k} \alpha_{k-1}(X, Y) d X^{2}+2(k+2) X^{k} a_{k}(Y) d X^{2}+\mathcal{O}\left(X^{k+1}\right)$

So picking $a$ appropriately, we eliminate the error at order $k$.

So we have shown that we can construct $\psi_{r}$ for all $r$. Now as $\psi_{r}$ fixes the boundary to order $k+1$, the first $k$ terms of the Taylor series of $\phi_{r}$ will be independent of $r$ for $r>k$. Using the Borel lemma, we therefore choose $\psi$ to have these values as its Taylor series.

By construction $\psi$ has the requisite properties.

To what extent is this model unique? In [3], for scattering metrics it is noted that $x$ is determined modulo $x^{2}$ by the metric and that the $x^{2}$ term in the Taylor series is arbritrary but that all succeeding terms are forced by the metric. In [2], for zero metrics it is observed that the term $x$ can be replaced by $c(y) x$ with an arbritrary 
positive smooth function $c$ but all succeeding terms are determined. What occurs with exact $b$-metrics?

If we put $x=c(y) X$, we get an additional term, $2 X^{-1} c^{-1} \frac{\partial c}{\partial y} d y d X$. This has to be zero so we conclude that $c$ is constant. So the lead term of $x$ is determined up to constants by the smooth structure and by the metric.

Now suppose $x=X+e(y) X^{2}$. We then gain an extra term, $2 X^{-1} e(Y) d X^{2}$. This has to be zero so $e=0$.

All further terms have been seen to be forced above. So we conclude that modulo terms vanishing to infinite order at the boundary, the choice of $x$ modulo a constant scalar is unique. This is a greater amount of rigidity than in the zero and scattering cases where one term of the Taylor series was arbritrary.

\section{REFERENCES}

[1] Tanya Christiansen, Spectral Theory for Manifolds with Asymptotically Cylindrical Ends, J. Funct. Anal. 131 (1995), No 2, 499-530

[2] M.S. Joshi, A. Sá Barreto, Inverse Scattering on Asymptotically Hyperbolic Manifolds, to appear in Acta Math.

[3] M.S. Joshi, A. Sá Barreto, Recovering Asymptotics of Metrics from Fixed Energy Scattering Data, Invent. Math. 137 (1999), 127-143 CMP 99:16

[4] R.B. Melrose, Geometric Scattering Theory, Cambridge University Press 1995 MR 96k:35129

[5] R.B. Melrose The Atiyah-Patodi-Singer Index Theorem, Research Notes in Mathematics 4, A.K. Peters Ltd, Wellesley, MA, 1993 MR 96g:58180

Department of Pure Mathematics and Mathematical Statistics, University of CamBridge, 16 Mill Lane, Cambridge CB2 1SB, England, United Kingdom

E-mail address: joshi@dpmms.cam.ac.uk

Current address: NatWest Group Risk, 135 Bishopsgate, London EC2M 3UR, England, United Kingdom 